\title{
Towards HIV-1 remission: potential roles for broadly neutralizing antibodies
}

\author{
Ariel Halper-Stromberg ${ }^{1}$ and Michel C. Nussenzweig ${ }^{1,2}$ \\ 'Laboratory of Molecular Immunology, The Rockefeller University, New York, New York, USA. ${ }^{2}$ Howard Hughes Medical Institute, New York, New York, USA.
}

\begin{abstract}
Current antiretroviral drug therapies do not cure HIV-1 because they do not eliminate a pool of long-lived cells harboring immunologically silent but replication-competent proviruses - termed the latent reservoir. Eliminating this reservoir and stimulating the immune response to control infection in the absence of therapy remain important but unsolved goals of HIV-1 cure research. Recently discovered broadly neutralizing antibodies (bNAbs) exhibit remarkable breadth and potency in their ability to neutralize HIV-1 in vitro, and recent studies have demonstrated new therapeutic applications for passively administered bNAbs in vivo. This Review discusses the roles bNAbs might play in HIV-1 treatment regimens, including prevention, therapy, and cure.
\end{abstract}

\section{Introduction}

Combination antiretroviral therapy (ART) suppresses viremia and extends the life span of infected individuals, but the daily burden of ART and its associated toxicities make an HIV cure highly desirable. Current therapies are not curative because they do not eliminate a pool of long-lived cells harboring immunologically silent but replication-competent proviruses - termed the latent reservoir. This reservoir is refractory to ART and, although not proven, is believed to be the source of viral rebound shortly after discontinuation of standard therapy (1). However, the precise anatomic location of this persistent reservoir remains unknown, and recent studies suggest that viral rebound following treatment interruption may arise from many cells at multiple sites (2). Completely eliminating this reservoir, referred to as sterilizing cure, or stimulating the immune response to control infection in the absence of therapy, referred to as functional cure, are the primary aims of cure research. However, both strategies present unresolved challenges, and the potential characteristics of a broadly applicable cure regimen remain unclear. While many approaches, ranging from gene therapy to immune modulation, are being investigated, this Review focuses on the roles that broadly neutralizing antibodies (bNAbs) might play in therapy and how these roles could extend to cure strategies.

In the decades after HIV-1 was discovered, the identification of anti-HIV-1 bNAbs with extensive breadth and potency was difficult; however, technological advances in antibody cloning from single B cells and microneutralization assays have led to the recent discovery of hundreds of naturally arising bNAbs, referred to as "next-generation" bNAbs (3-10). Some of these bNAbs exhibit remarkable breadth and potency in vitro, and many of these bNAbs can prevent infection in animal models when passively administered at low doses before challenge (11-14). Therefore, it is widely believed that a vaccine that could

Conflict of interest: The authors have declared that no conflict of interest exists. Reference information: J Clin Invest. 2016;126(2):415-423. doi:10.1172/JCI80561. elicit such antibodies would be protective against infection, and the development of such a vaccine remains an important goal. However, most next-generation bNAbs only arise after at least a year of active infection $(15,16)$, and many show a number of unusual features, such as high levels of somatic hypermutation, long heavy chain complementarity-determining region 3 regions, and restricted germline use. Together, these features suggest that it will be difficult if not impossible to elicit bNAbs against HIV-1 by standard vaccine approaches. The uncertainties associated with a vaccine have led us, and others, to explore the use of bNAbs in passive transfer experiments for HIV-1 prevention, therapy, and eradication.

\section{bNAb passive immunotherapy}

Next-generation bNAbs have rapidly progressed through preclinical and clinical studies, with therapeutic efficacy being demonstrated first in humanized mice $(17,18)$, then in nonhuman primates (NHPs) $(19,20)$, and finally in humans (21) - all in the span of three years (refs. 17, 19-21 and Table 1). Next-generation bNAb monotherapy $(20 \mathrm{mg} / \mathrm{kg})$ produced transient drops in viral load of 0.23 to $1.5 \log _{10}$ copies in humanized mice, with rapid selection for viruses containing mutations within the viral envelope protein (Env) that confer resistance to the corresponding bNAbs (17). For each efficacious bNAb tested, confirmed escape mutations were limited to just 1 to 3 residues, highlighting the narrow escape routes from these potent reagents. A combination of three antibodies (PG16, 10-1074, and 3BNC117) targeting three distinct epitopes prevented viral escape and suppressed viremia beneath detection in humanized mice as long as antibody concentrations were maintained above 1 to $10 \mu \mathrm{g} / \mathrm{ml}(18)$. When ART was administered to humanized mice to suppress viral load before bNAb therapy, a single bNAb was able to maintain viral suppression in most cases (18).

In light of successful antibody therapy in humanized mice, bNAbs were tested in NHPs. Before these studies, NHPs were believed to pose a significantly tougher challenge to bNAb therapy relative to humanized mice, because infected NHPs contain orders 
Table 1. Summary of next-generation bNAb immunotherapy results

$\begin{array}{lcc}\text { Model } & \begin{array}{c}\text { Challenge } \\ \text { virus }\end{array} & \text { bNAbs tested } \\ \begin{array}{l}\text { Humanized } \\ \text { mouse }^{A}\end{array} & \text { HIV-1 } \text { YU2 }_{\text {Y }} & 3 B N C 117, \\ & & \text { PG16, PGT128, } \\ & & 10-1074, \\ & 45-46^{654 W} \\ \text { Rhesus } & \text { SHIV }_{\text {SF162P3 }} & 3 B N C 117, \\ \text { macaque } & & \text { PGT121, b12 }\end{array}$

$\begin{array}{lcc}\begin{array}{l}\text { Rhesus } \\ \text { macaque }\end{array} & \text { SHIV }_{\text {AD8 }} & \begin{array}{l}3 B N C 117, \\ 10-1074\end{array} \\ & & \\ \text { Human } & \begin{array}{c}\text { Patient virus } \\ \text { (clade B) }\end{array} & \text { 3BNC117 }\end{array}$

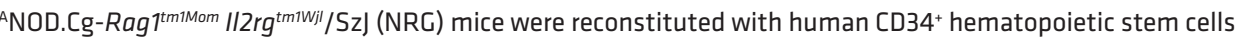

\section{Combination therapy result}

Viral escape by mutation at restricted residues that abrogate bNAb binding

Viral suppression when plasma antibody levels $>10 \mu \mathrm{g} / \mathrm{ml}$ in $55 \%$ of individuals measured to 1.1 logs

2 of 2 3BNC117-treated animals achieved viral suppression, with viral rebound occurring without apparent resistance mutations when antibody levels dropped below $10 \mu \mathrm{g} / \mathrm{ml}$; 2 of $210-1074$-treated animals achieved viral suppression, with viral rebound occurring with resistance mutations at antibody-binding residues

Transient decline in plasma viremia of 0.8 to $2.5 \log _{10}$, with $>5$-fold decline in viral sensitivity to 3BNC117
Viral suppression achieved in 4 of 5 animals treated with 3BNC117 and 10-1074 combination therapy, with viral rebound occurring when monoclonal antibody levels dropped below $10 \mu \mathrm{g} / \mathrm{ml}$

\begin{abstract}
Viral suppression in 12 of 14 animals, with viral rebound occurring without detectable resistance mutations when monoclonal antibody levels dropped below $1 \mu \mathrm{g} / \mathrm{ml}$ 4 animals having rebound viremia when PGT levels declined to undetectable levels; 4 of 4 3BNC117-treated animals showed transient declines in plasma viremia of 0.2

4 of 4 PGT121-treated animals achieved viral suppression,

2
\end{abstract}

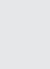

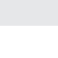

(1)

Untested

21

References

17

19

20

Untested 21 
A
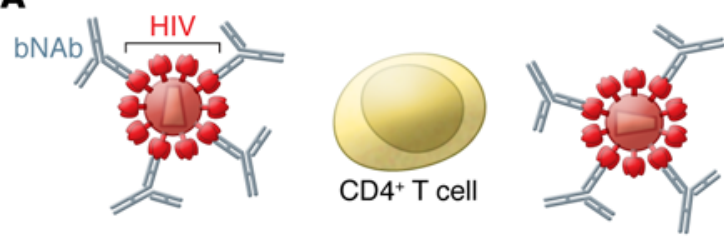

B
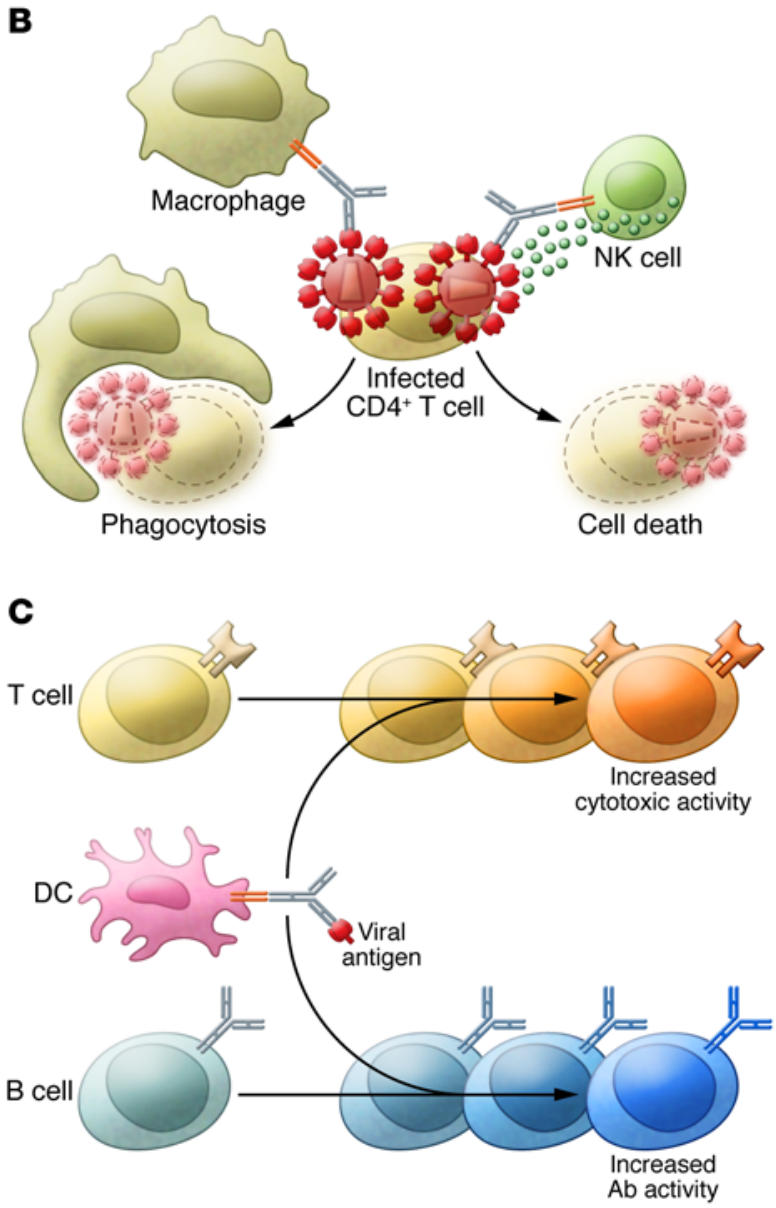

Direct binding to virions not only neutralizes the virus before entry but also raises the possibility of accelerating viral clearance from plasma. Indeed, the half-life of cell-free virus in the plasma, as measured by viral RNA, was 2.3-fold shorter in macaques that had circulating Env-binding antibodies compared with that in macaques with no virus-specific antibodies (33). When infectivity was measured rather than viral RNA, there were no infectious particles detected within 10 to 15 minutes of virus infusion in 4 of 5 macaques with Env-binding antibodies, suggesting that Env-binding antibodies neutralize virions in plasma even before they are cleared. It is possible that bNAbs, which are far more potent than the pooled IgG used in this study, could exhibit even greater effects on virions (Figure 1).

Overall, cell-free virus is cleared from plasma very rapidly, even in the absence of antibodies $(33,34)$, but bNAbs may be able to clear viruses in tissues as well. This could prove clinically important, given the high levels of viral spread within lymphoid tissue, which has high densities of target cells. Virions are likely to contact target cells rapidly in these tissues and gain cellular entry within an hour of initial contact (35).
Figure 1. Potential immune effector functions of bNAbs. (A) bNAbs can prevent infection by directly neutralizing cell-free virions, which prevents infection and accelerates viremia decline through antibody-mediated viral clearance. (B) bNAbs can potentially bind to infected cells and mediate antiviral activity through Fc-FcR interactions that engage NK cells for ADCC or macrophages for phagocytosis and cellular destruction. (C) Immune complexes formed by bNAbs bound to viral antigen can be taken up by dendritic cells and may stimulate host adaptive immune responses, including increased antiviral cytotoxic T cell activity and B cell maturation.

\section{Fc-mediated effects of antibodies}

In addition to their ability to bind and neutralize pathogens directly, antibody molecules also engage potent immune effector mechanisms (Figure 1). Antibodies can bind complement to stimulate cell lysis by the classical pathway, they can engage $\mathrm{Fc}$ receptors (FcRs) on immune cells to clear infected cells by antibody-dependent cellular cytotoxicity (ADCC), and antibodies can form immune complexes that activate dendritic cells to enhance antigen presentation (36). Which, if any, of these immune effector mechanisms plays a significant role in passive immunotherapy against HIV- 1 is not known. However, the potent effects of antibodies in therapy as well as the results of the RV144 vaccine trial have renewed interest in the role of ADCC in the context of HIV infection. The immune correlates analysis of the RV144 trial showed that nonneutralizing antibodies targeting the V2 loop protected against infection, suggesting that ADCC played a role in preventing viral acquisition (37). Subsequently, mAbs that mediated increased infected cell killing in vitro were isolated from RV144 vaccinees (38); however, the data supporting an antibody-mediated clearance effect on virus or cells in humans remain indirect.

Todate, directevidence for Fc-mediated effects in HIV infection comes entirely from animal models. To determine whether bNAbs are effective as postexposure prophylaxis, we infected humanized mice with $\mathrm{HIV}-1_{\mathrm{YU}}$ and then initiated ART or combination bNAbs 96 hours after infection. While plasma viremia remained undetectable in both treatment groups during ART or bNAb therapy, a significantly higher proportion of ART-treated mice showed rebound plasma viremia compared with antibody-treated mice when therapy was discontinued. This suggests that, although ART was able to block viral replication, bNAbs may have reduced the pool of cells capable of producing viral rebound or maintained durable suppression because of their long half-life (39).

To determine whether Fc-mediated effects contributed to the antiviral function of bNAbs in humanized mice, we tested the same combination of bNAbs carrying point mutations in the Fc regions that abrogate binding to mouse and human FcRs and complement. While plasma viremia was suppressed comparably to WT bNAbs during Fc mutant bNAb therapy, viral rebound in mice receiving Fc mutant bNAbs occurred significantly earlier following the last bNAb injection (39). This result suggested that bNAbs lacking the ability to engage $\mathrm{Fc}$ receptor-expressing (FcR-expressing) cells were capable of blocking new infections, but Fc-mediated cellclearing effects are required for the full potency of bNAbs.

Bournazos and colleagues extended these findings to preexposure prophylaxis in an immunocompetent mouse model for HIV entry. They found that bNAbs engineered to express the murine IgG2a Fc subclass, which preferentially binds murine activating FcRs (mFcrRI and mFc $\gamma$ RIV) relative to inhibitory FcRs, were 
A
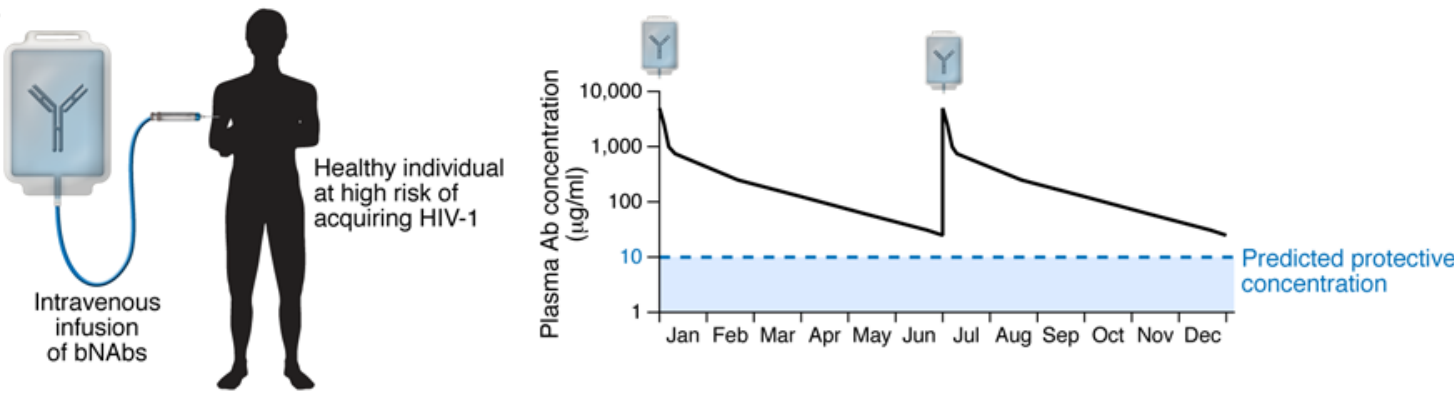

B

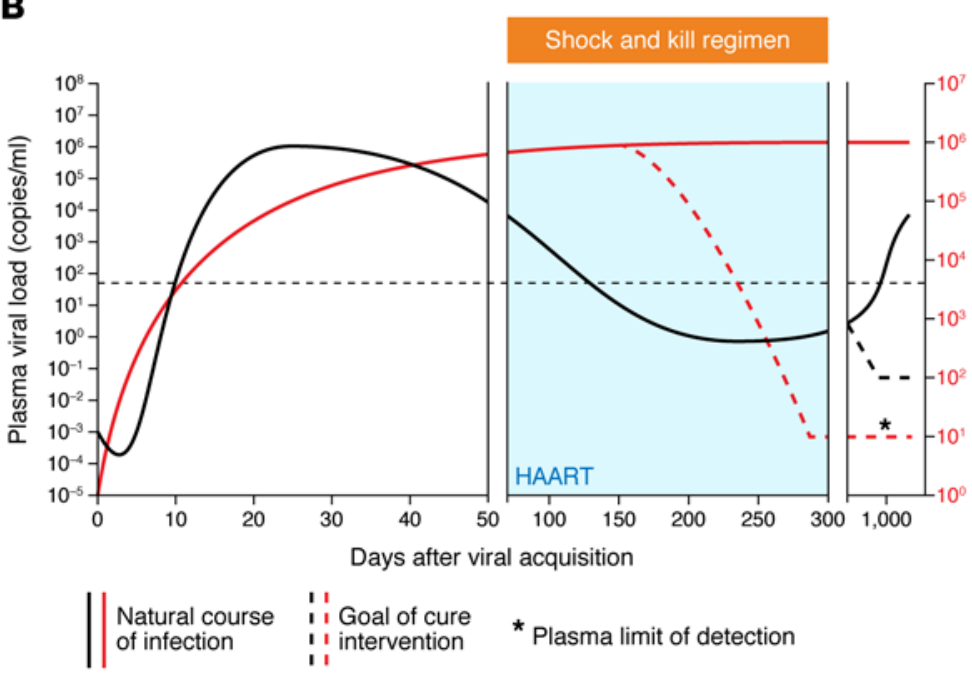

C

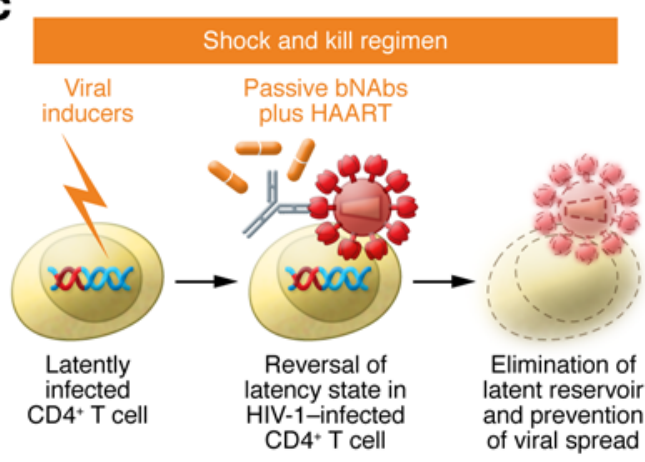

Figure 2. bNAbs in prevention and cure regimens. (A) The potency and long in vivo half-lives of bNAbs may enable biannual passive administrations to protect against infection in healthy individuals at high risk of HIV-1 exposure. In the schematic shown, uninfected individuals can receive intravenous infusions of long-acting monoclonal bNAbs for prophylactic protection against HIV-1 acquisition. The plot shows the estimated peak plasma bNAb levels following infusion and extrapolates the decay based on bNAb pharmacokinetics measured in human pilot studies (21). The predicted protective plasma concentration is estimated based on NHP studies $(14,19)$. (B) Schematic of the natural course of HIV-1 viremia (black) and latent reservoir stability (red) with the current standard of care, highly active ART (HAART) (solid lines). The goal of the shock and kill intervention is to clear the latent reservoir and prevent viral rebound following therapy discontinuation (dotted line). (C) The shock and kill regimen indicated in $\mathbf{B}$ is shown schematically.

significantly more effective at preventing viral entry than their IgG1 Fc subclass counterparts (40). This effect was abolished in $\mathrm{F} c \gamma \mathrm{R} \alpha^{\mathrm{NULL}}$ mice. Similar findings were reported in macaques. In models using both single high-dose viral challenge and repeated low-dose viral challenges, prophylactic treatment with WT b12 antibody markedly reduced viral acquisition relative to a variant of b12 that could not bind FcRs (L234A/L235A) $(12,41)$.

These findings show that Fc-mediated effects are important for both preventing and clearing infection. However, these studies did not produce direct evidence that productively infected cells were cleared by bNAbs. Rather, indirect measurements, such as time to rebound or onset of peak viremia, were reported. If bNAbs directly clear infected cells, viral clearance should be accelerated relative to ART, since new rounds of replication are blocked and infected cells are also cleared. These kinetic measurements are difficult in humanized mice because of the small number of infected cells, but such measurements were attempted in two separate macaque studies. In one study, Barouch and colleagues reported that plasma viremia declined at an accelerated rate in PGT121treated macaques $(0.383 \mathrm{log} /$ day $)$ (19) compared with that in ARTtreated macaques (0.229 $\mathrm{log} /$ day) (42) or ART-treated humans (0.264 log/day) (43). However, Shingai and colleagues reported that an infusion of a combination of 3BNC117 and 10-1074 led to a plasma viremia decline of $0.225 \mathrm{log} /$ day (20). Thus, the data from macaque studies are inconclusive in terms of viral clearance rates.

Overall, the evidence suggests that eliminating $\mathrm{Fc}$-mediated function impairs the protective antiviral activity of bNAbs, but it is unclear whether enhancing ADCC activity improves protective efficacy. A nonfucosylated variant of b12 that had enhanced viral inhibitory and killing abilities in vitro failed to improve protection efficacy in vivo relative to WT b12 (44). Thus, definitive evidence for ADCC remains elusive. The development of sensitive assays to detect infected cells is likely necessary to clarify the role of bNAbs in these processes. While ADCC could impact the speed with which viremia becomes undetectable in patients treated with passive bNAbs, ADCC by itself is not sufficient for achieving viral remission. Boosting the immune system's ability to control HIV and interfering with the latent reservoir are also necessary to achieve cure.

\section{bNAbs and the immune system}

The finding that single bNAbs were able to suppress viremia without escape in macaques - a result rarely seen in immunocompromised mice - suggests differences between the two models or the different challenge viruses used. Klein and colleagues investigated 
whether part of the difference was attributable to the macaques' intact humoral immune system. They showed that sera taken from SHIV $_{\mathrm{ADs}}$-infected NHPs was more potent against viruses carrying signature escape mutations to bNAbs than the corresponding primary WT isolates (45), suggesting that endogenously produced antibodies could keep viruses harboring bNAb escape mutations in check. Going forward, the critical question is whether passively administered bNAbs are capable of preventing viral escape in the presence of endogenous IgG. It is especially important to determine whether infected individuals whose viremia has been suppressed for protracted periods with ART harbor HIV-specific antibodies capable of neutralizing viruses carrying bNAb escape mutations. The answer should be borne out by ongoing clinical trials in which there is an opportunity to investigate the synergy between bNAbs and endogenously produced antibodies.

In addition to synergizing with endogenous antibodies, passive mAbs may stimulate the immune system (Figure 1). Antibody therapy in macaques was shown to decrease expression of the exhaustion marker programmed death- 1 on $\mathrm{CD}^{+}$and $\mathrm{CD} 8^{+}$ $\mathrm{T}$ cells and increase the ability of cytotoxic T lymphocytes (CTLs) to suppress viral replication ex vivo (19). Modest increases in neutralizing titers were seen in some macaques, suggesting that the passive mAbs may have stimulated endogenous B cell responses. Immune complexes of antibody-bound viral particles are highly effective at stimulating dendritic cell maturation and immune responses and may provide a mechanism to explain the increased neutralizing titers (36). Although additional studies are necessary to clarify the magnitude, generality, and underlying mechanisms of these effects, the potential to augment anti-HIV-1 immune responses suggests that passive bNAbs should be explored in functional cure strategies. However, the utility of bNAbs in sterilizing cure requires targeting of the latent reservoir, which likely requires interventions in addition to bNAbs.

\section{Targeting the latent reservoir}

Currently available therapies do not clear the latent reservoir. Studies in macaques and humans treated with ART shortly after infection demonstrated that an ART-refractory reservoir capable of producing rebound viremia is established as early as 24 to 72 hours after exposure $(42,46-48)$. Furthermore, the size of the latent reservoir is stable over time, even with continued ART (49). Although the complete cellular composition of the clinically relevant reservoir remains a subject of debate, resting memory $\mathrm{CD} 4^{+} \mathrm{T}$ cells are certainly an important component. An estimated $10^{4}-10^{7}$ latently infected resting memory $\mathrm{CD} 4^{+} \mathrm{T}$ cells are found within an individual (50). The exact half-life of these cells is difficult to measure accurately or precisely because of the limitations in the assays available to measure the reservoir. As a result, the average half-life of latently infected cells has been reported to vary from 6 to 44 months $(49,51-54)$, with an even wider range reported for individual patients.

Irrespective of the reservoir's precise half-life, latently infected cells remain in the infected host for prolonged periods of time, and the mechanisms by which they persist are unknown. Intensifying ART from the normal three-drug regimens to five-drug regimens fails to measurably reduce the size of the latent reservoir $(55,56)$. Thus, interventions beyond ART are necessary to clear the latent reservoir. The leading proposed strategy, termed "shock and kill," uses either immunologic or pharmacologic interventions to administer a "shock" that activates HIV replication within latently infected cells in the presence of ART. The objective is to induce viral transcription and activate productive infection, with the idea that productively infected cells die by cytopathic effects or immunologic attack (Figure 2). ART is continued during this process to prevent the spread of infection (57). This strategy was first tested clinically by administering IL-2 and/or IFN- $\gamma$ with the hope of stimulating T cell activation and HIV-1 transcription (58-60). However, cytokine therapy was too toxic for systematic evaluation, and the data indicated little or no impact on the latent reservoir.

More recent efforts have focused on histone deacetylase inhibitors (HDACis), which stimulate HIV transcription from latently infected cells, without inducing global $\mathrm{T}$ cell activation and cytokine storm $(61,62)$. The HDACis vorinostat, panobinostat, and romidepsin were shown to stimulate HIV transcription in latently infected cells obtained from ART-suppressed patients in vivo (63-65), as measured by cell-associated unspliced Gag transcripts. However, clinical studies showed no effect on latent reservoirs, as measured by quantitative viral outgrowth assays or the time to rebound after analytic treatment interruption $(65,66)$.

Why there was little or no measurable effect on the reservoir after HDAC inhibition despite increased Gag transcription is not known. However, the levels of transcription were low and, perhaps, insufficient to induce cell death. Consistent with this idea, Shan and colleagues showed that cells treated with vorinostat failed to die at the normal rate, despite reactivating an HIV-1 indicator gene, suggesting that vorinostat-reactivated cells do not die by cytopathic effects alone (67). Additionally, vorinostat induces transcription in only a small subset of latent cells $(68,69)$, making it unlikely that the subsequent disappearance of reactivated cells from the reservoir could be measured accurately. In total, the available data highlight the need for advances in both the "shock" and "kill" arms of the approach.

\section{bNAbs and "shock and kill"}

The role of bNAbs in "shock and kill" is linked to their ability to neutralize virions released from reactivated latent cells, kill infected cells, and access immune-privileged anatomic sites. Chun and colleagues showed that bNAbs bind and neutralize virions released from ART-suppressed patients' resting $\mathrm{CD}^{+}{ }^{+} \mathrm{T}$ cells that are stimulated with CD3/CD28 antibodies (32). Because most proviruses archived in the latent reservoir of ART-treated patients are resistant to autologous CTL responses (70) and HDACis such as romidepsin and panobinostat may impair the function of virus-specific CTLs (71), relying on an infected individual's CD8 ${ }^{+}$ $\mathrm{T}$ cells to kill reactivated cells likely requires therapeutic vaccination. In contrast, passive immunotherapy may block viral spread and can be individually tailored based on the resistance and sensitivity profiles of inducible viruses from a patient's latent reservoir.

In addition to neutralizing virions released from reactivated cells, bNAbs may be able to kill reactivated cells directly. To function as effective killing agents, bNAbs have to bind reactivated cells and recruit cytolytic innate immune cells. bNAbs have been shown to bind Env on the surface of the latency reporter cell lines $\mathrm{ACH} 2$ and $\mathrm{J} 89$ stimulated with panobinostat 
and romidepsin (72), but to date, bNAbs have not been shown to bind reactivated primary $\mathrm{CD} 4^{+} \mathrm{T}$ cells. Nevertheless, it is likely that reactivated cells express Env in response to inducing agents because viral particles bud and are detectable in supernatant $(73,74)$. New pharmacologic agents that induce detectable plasma viremia in patients who were previously viremia suppressed on ART could enable bNAbs to find and kill reactivated cells. GS-9620, a TLR7 agonist, was reported to induce detectable plasma viremia in ART-suppressed macaques (75); romidepsin was reported to do the same in humans (76). Determining the proportion of latent cells that are reactivated is critical for predicting whether these candidate inducers are likely to significantly impact the reservoir in patients.

The theoretical contribution of bNAbs to HIV eradication efforts is based on the general mechanisms of antibody function. This idea has only been tested in vivo in humanized mice treated with a combination of three bNAbs (PG16, 10-1074, and 3BNC117) and three viral inducers [(I-BET151, vorinostat, and anti-cytotoxic $\mathrm{T}$ lymphocyte antigen-4 (anti-CTLA4)]. In that experiment, the combination of inducers and bNAbs reduced the proportion of mice showing viral rebound when antibody levels decayed, whereas treatment with antibodies alone or antibodies plus a single inducer did not reduce the proportion of mice with rebound viremia (39). The results highlight the likely necessity of a combination of mechanistically independent inducers to achieve sufficient viral transcription and viral spike cell surface expression to allow antibodies to exert their effects (77). However, the inducer combination and dosing schedule were not optimized in that study, and efforts to identify optimal combinations of inducers (78) as well as technologies to effectively administer them (79) will be paramount for maximizing the ability of bNAbs to kill latent cells. Additionally, limitations of the mouse model required that viral suppression before administration of inducers was limited to several weeks as opposed to months in human trials. Furthermore, the size of the reservoir in mice is much smaller than in humans, and the composition of the reservoir is likely to be different. Clinical trials testing inducer combinations with bNAbs are required to see how this approach translates to humans.

\section{bNAbs and anatomical sanctuaries}

In addition to direct binding to infected cells, the role of bNAbs in HIV cure is attributable to their widespread tissue penetrance where sufficient concentrations of ART may be limiting. While the existence of anatomic sanctuaries in which drug levels do not reach fully suppressive levels is debatable, some tissues clearly support higher levels of viral spread than others, such as gut-associated lymphoid tissue and lymph nodes (80-82). It is possible that direct cell-to-cell spread of HIV in these tissues is less sensitive to ART because of the high multiplicity of infection that occurs in these tissues (82). In contrast, antibodies are found in high concentrations in interstitial fluids, and some bNAbs can prevent cellto-cell viral spread in vitro $(83,84)$.

Certain cellular subsets may preferentially shield infected cells from autologous immune responses but are likely to remain sensitive to bNAbs. Fukazawa and colleagues showed that typical progressor monkeys exhibit widespread SIV infection across many
$\mathrm{CD}^{+} \mathrm{T}$ cell subtypes, whereas elite controller monkeys with potent antiviral $\mathrm{CD}^{+} \mathrm{T}$ cell responses harbor infection predominantly in $\mathrm{CD}^{+}$follicular helper $\mathrm{T}$ cells $\left(\mathrm{T}_{\mathrm{FH}}\right)$ in B cell follicles (85). Depletion of $\mathrm{CD}^{+} \mathrm{T}$ cells in elite controller monkeys causes reversible redistribution of SIV into non- $\mathrm{T}_{\mathrm{FH}}$ cells, suggesting that the $\mathrm{T}_{\mathrm{FH}}$ cells within the follicles are protected from $\mathrm{CD} 8^{+} \mathrm{T}$ cell-mediated clearance. Unlike $\mathrm{CD} 8^{+} \mathrm{T}$ cells, antibodies are not anatomically restricted from $B$ cell follicles, and direct testing of effects of bNAbs on infected $\mathrm{T}_{\mathrm{FH}}$ cells should be investigated.

While passively infused antibodies have widespread tissue distribution (13), a potential caveat is that they have poor penetrance into the brain. The brain penetrance of anti-HIV-1 bNAbs has not been measured in humans, but other human IgG1 class antibodies have been reported to achieve 500 to 1,000 times lower concentrations in the cerebrospinal fluid relative to serum $(86,87)$. Although the role of viral replication and viral reservoirs in the central nervous system and other potential sanctuaries such as reproductive organs remains unclear, the effects of bNAbs in these tissues require further investigation.

\section{bNABs and prevention}

In addition to playing a role in eradication, bNAbs offer promise as prophylaxis though passive immunization (Figure 2). The initial phase I clinical data showing that 3BNC117 is effective and safe in humans support testing of this approach (21). As a result of their potency, low concentrations of bNAbs are able to prevent mucosal transmission in high-dose challenge models in humanized mice and macaques $(13,14,88)$. Probit analysis of a large cohort of macaques treated with several different antibodies indicated that the amount of antibody needed for $50 \%$ protection against intrarectal infection in macaques corresponds to serum levels of 100 times the $\mathrm{IC}_{50}$ of the antibody, as determined by in vitro infection assay of TZM-bl cells (13). However, humans infected by mucosal routes are typically exposed to low doses of HIV-1, and existing data indicate that the protective bNAb levels may be lower than that required for high-dose challenge (11).

Because bNAbs are naturally arising, fully human products, they can have long half-lives on the order of 2 to 3 weeks. Based on the serum antibody levels reached by passive infusions of 3BNC117 and VRC01 in humans as well as the 17.5 day half-life of 3BNC117 in uninfected individuals (21), it appears that a serum level of 100 times the $\mathrm{IC}_{50}$ is achievable for long periods of time with infrequent infusions, making a biannual passive antibody injection within the realm of possibility for protection (Figure 2). As an alternative to $\mathrm{mAb}$ infusions, adeno-associated viral vectors expressing bNAbs have also been shown to provide lasting protection against HIV infection $(89,90)$. Clinical trials testing these concepts are necessary to determine how often antiantibodies that reduce bNAb half-life develop and interfere with protection.

\section{Perspectives}

Despite the rapid progress of HIV antibody therapy, many challenges remain for eradication. The greatest obstacle is likely the identification of agents that sufficiently reactivate latent cells. With regards to bNAbs, determining which effector cells mediate ADCC most effectively - and ensuring that these cells are optimally targeted - is critical to maximizing therapeutic effects. Additionally, 
determining which subclasses of antibodies exert the strongest effects in certain tissues could inform antibody-engineering efforts.

Finally, many potential modifications can enhance the antiviral functions of antibodies in vivo, such as mutations that enhance half-life, FcR binding, and potency (40, 91-93). Bispecific antibodies that express two different Fab regions have shown increased potency in vitro $(94,95)$, and coupling antibodies to immunotoxins to enhance killing of infected cells is being explored in vivo (96). Both strategies remain active areas of investigation. Considering the disappointments from HIV vaccine and cure trials performed to date, we must move forward with guarded optimism and a focus on the basic science behind HIV antibody functions. Hopefully the lessons learned will not only improve HIV treatment, but also accelerate development of mAb therapies for other highly mutable pathogens.

\section{Acknowledgments}

We thank all members of the Nussenzweig laboratory for critical reading of the manuscript. This work was supported by NIH Medical Scientist Training Program grant T32GM07739 to the Weill Cornell/Rockefeller/Sloan-Kettering Tri-Institutional MD-PhD Program (to A. Halper-Stromberg), NIH F30 grant AI10983002 (to A. Halper-Stromberg), NIH Center for HIV/AIDS Vaccine Immunology and Immunogen Discovery (AI100663-04 to M.C. Nussenzweig), and National Institute of Allergy and Infectious Diseases grant AI100148-03.

Address correspondence to: Michel C. Nussenzweig, Laboratory of Molecular Immunology, 1230 York Ave., Box 220, New York, New York 10065-6399, USA. Phone: 212.327.8067; E-mail: nussen@ rockefeller.edu.
1. Davey RT Jr, et al. HIV-1 and T cell dynamics after interruption of highly active antiretroviral therapy (HAART) in patients with a history of sustained viral suppression. Proc Natl Acad Sci U S A. 1999;96(26):15109-15114.

2. Rothenberger MK, et al. Large number of rebounding/founder HIV variants emerge from multifocal infection in lymphatic tissues after treatment interruption. Proc Natl Acad Sci U S A. 2015;112(10):E1126-E1134.

3. Walker LM, et al. Broad and potent neutralizing antibodies from an African donor reveal a new HIV-1 vaccine target. Science. 2009;326(5950):285-289.

4. Wu X, et al. Rational design of envelope identifies broadly neutralizing human monoclonal antibodies to HIV-1. Science. 2010;329(5993):856-861.

5. Scheid JF, et al. Sequence and structural convergence of broad and potent HIV antibodies that mimic $\mathrm{CD} 4$ binding. Science. 2011;333(6049):1633-1637.

6. Walker LM, et al. Broad neutralization coverage of HIV by multiple highly potent antibodies. Nature. 2011;477(7365):466-470.

7. Mouquet $\mathrm{H}$, et al. Complex-type $\mathrm{N}$-glycan recognition by potent broadly neutralizing HIV antibodies. Proc Natl Acad Sci U S A. 2012;109(47):E3268-E3277.

8. Huang J, et al. Broad and potent neutralization of HIV-1 by a gp 41-specific human antibody. Nature. 2012;491(7424):406-412.

9. Liao HX, et al. Co-evolution of a broadly neutralizing HIV-1 antibody and founder virus. Nature. 2013;496(7446):469-476.

10. Huang J, et al. Broad and potent HIV-1 neutralization by a human antibody that binds the gp 41gp120 interface. Nature. 2014;515(7525):138-142.

11. Mascola JR, et al. Protection of Macaques against pathogenic simian/human immunodeficiency virus $89.6 \mathrm{PD}$ by passive transfer of neutralizing antibodies. JVirol. 1999;73(5):4009-4018.

12. Hessell AJ, et al. Effective, low-titer antibody protection against low-dose repeated mucosal SHIV challenge in macaques. Nat Med. 2009;15(8):951-954.

13. Moldt B, et al. Highly potent HIV-specific antibody neutralization in vitro translates into effective protection against mucosal SHIV challenge in vivo. Proc Natl Acad Sci U S A. 2012;109(46):18921-18925.

14. Shingai M, et al. Passive transfer of modest titers of potent and broadly neutralizing anti-HIV monoclonal antibodies block SHIV infection in macaques. JExp Med. 2014;211(10):2061-2074.

15. Mikell I, et al. Characteristics of the earliest cross-neutralizing antibody response to HIV-1. PLoS Pathog. 2011;7(1):e1001251.

16. Gray ES, et al. The neutralization breadth of HIV-1 develops incrementally over four years and is associated with $\mathrm{CD} 4^{+} \mathrm{T}$ cell decline and high viral load during acute infection. JVirol. 2011;85(10):4828-4840.

17. Klein F, et al. HIV therapy by a combination of broadly neutralizing antibodies in humanized mice. Nature. 2012;492(7427):118-122.

18. Horwitz JA, et al. HIV-1 suppression and durable control by combining single broadly neutralizing antibodies and antiretroviral drugs in humanized mice. Proc Natl Acad Sci U S A. 2013;110(41):16538-16543.

19. Barouch DH, et al. Therapeutic efficacy of potent neutralizing HIV-1-specific monoclonal antibodies in SHIV-infected rhesus monkeys. Nature. 2013;503(7475):224-228.

20. Shingai M, et al. Antibody-mediated immunotherapy of macaques chronically infected with SHIV suppresses viraemia. Nature. 2013;503(7475):277-280.

21. Caskey M, et al. Viraemia suppressed in HIV-1infected humans by broadly neutralizing antibody 3BNC117. Nature. 2015;522(7557):487-491.

22. Poignard $P$, et al. Neutralizing antibodies have limited effects on the control of established HIV-1 infection in vivo. Immunity. 1999;10(4):431-438.

23. Trkola A, et al. Delay of HIV-1 rebound after cessation of antiretroviral therapy through passive transfer of human neutralizing antibodies. Nat Med. 2005;11(6):615-622.

24. Mehandru S, et al. Adjunctive passive immunotherapy in human immunodeficiency virus type 1-infected individuals treated with antiviral therapy during acute and early infection. J Virol. 2007;81(20):11016-11031.

25. Pace CS, Fordyce MW, Franco D, Kao CY, Seaman MS, Ho DD. Anti-CD4 monoclonal antibody ibalizumab exhibits breadth and potency agains HIV-1, with natural resistance mediated by the loss of a V5 glycan in envelope. J Acquir Immune Defic Syndr. 2013;62(1):1-9.

26. Trkola A, et al. Potent, broad-spectrum inhibition of human immunodeficiency virus type 1 by the CCR5 monoclonal antibody PRO 140.J Virol. 2001;75(2):579-588.

27. Norris D, et al. Phase 2 efficacy and safety of the novel entry inhibitor, TNX-355, in combination with optimized background regimen (OBR). AIDS 2006 - XVI International AIDS Conference; Abstract no. TUPE0058.

28. Jacobson JM, et al. Phase 2a study of the CCR5 monoclonal antibody PRO 140 administered intravenously to HIV-infected adults. Antimicrob Agents Chemother. 2010;54(10):4137-4142.

29. Toma J, et al. Loss of asparagine-linked glycosylation sites in variable region 5 of human immunodeficiency virus type 1 envelope is associated with resistance to CD4 antibody ibalizumab. JVirol. 2011;85(8):3872-3880.

30. Jacobson JM, et al. Antiviral activity of single-dose PRO 140, a CCR5 monoclonal antibody, in HIV-infected adults. J Infect Dis. 2008;198(9):1345-1352.

31. Jacobson JM, et al. Safety, pharmacokinetics, antiretroviral activity of multiple doses of ibalizumab (formerly TNX-355), an anti-CD4 monoclonal antibody, in human immunodeficiency virus type 1-infected adults. Antimicrob Agents Chemother. 2009;53(2):450-457.

32. Chun TW, et al. Broadly neutralizing antibodies suppress HIV in the persistent viral reservoir. Proc Natl Acad Sci U S A. 2014;111(36):13151-13156.

33. Igarashi $\mathrm{T}$, et al. Human immunodeficiency virus type 1 neutralizing antibodies accelerate clearance of cell-free virions from blood plasma. Nat Med. 1999;5(2):211-216.

34. Zhang L, et al. Rapid clearance of simian immunodeficiency virus particles from plasma of rhesus macaques. J Virol. 1999;73(1):855-860.

35. Arts EJ, Hazuda DJ. HIV-1 antiretroviral drug therapy. Cold Spring Harb Perspect Med. 2012;2(4):a007161.

36. Dhodapkar KM, et al. Selective blockade of inhibitory Fcgamma receptor enables human dendritic cell maturation with IL-12p70 production and 
immunity to antibody-coated tumor cells. Proc Natl Acad Sci U S A. 2005;102(8):2910-2915.

37. Haynes BF, et al. Immune-correlates analysis of an HIV-1 vaccine efficacy trial. $N$ Engl J Med. 2012;366(14):1275-1286.

38. Bonsignori M, et al. Antibody-dependent cellular cytotoxicity-mediating antibodies from an HIV-1 vaccine efficacy trial target multiple epitopes and preferentially use the VH1 gene family. J Virol. 2012;86(21):11521-11532.

39. Halper-Stromberg A, et al. Broadly neutralizing antibodies and viral inducers decrease rebound from HIV-1 latent reservoirs in humanized mice. Cell. 2014;158(5):989-999.

40. Bournazos S, Klein F, Pietzsch J, Seaman MS, Nussenzweig MC, Ravetch JV. Broadly neutralizing anti-HIV-1 antibodies require Fc effector functions for in vivo activity. Cell. 2014;158(6):1243-1253.

41. Hessell AJ, et al. Fc receptor but not complement binding is important in antibody protection against HIV. Nature. 2007;449(7158):101-104.

42. Whitney JB, et al. Rapid seeding of the viral reservoir prior to SIV viraemia in rhesus monkeys. Nature. 2014;512(7512):74-77.

43. Andrade A, et al. Three distinct phases of HIV-1 RNA decay in treatment-naive patients receiving raltegravir-based antiretroviral therapy: ACTG A5248. J Infect Dis. 2013;208(6):884-891.

44. Moldt B, et al. A nonfucosylated variant of the anti-HIV-1 monoclonal antibody b12 has enhanced Fc $\gamma$ RIIIa-mediated antiviral activity in vitro but does not improve protection against mucosal SHIV challenge in macaques. J Virol. 2012;86(11):6189-6196.

45. Klein F, et al. Enhanced HIV-1 immunotherapy by commonly arising antibodies that target virus escape variants. J Exp Med. 2014;211(12):2361-2372.

46. Chun TW, et al. Early establishment of a pool of latently infected, resting $\mathrm{CD} 4(+) \mathrm{T}$ cells during primary HIV-1 infection. Proc Natl Acad Sci U S A. 1998;95(15):8869-8873.

47. Tsai CC, et al. Prevention of SIV infection in macaques by (R)-9-(2-phosphonylmethoxypropyl)adenine. Science. 1995;270(5239):1197-1199.

48. Tsai CC, et al. Effectiveness of postinoculation (R)-9-(2-phosphonylmethoxypropyl) adenine treatment for prevention of persistent simian immunodeficiency virus SIVmne infection depends critically on timing of initiation and duration of treatment. J Virol. 1998;72(5):4265-4273.

49. Siliciano JD, et al. Long-term follow-up studies confirm the stability of the latent reservoir for HIV-1 in resting CD $4^{+}$T cells. Nat Med. 2003;9(6):727-728.

50. Chun TW, et al. Quantification of latent tissue reservoirs and total body viral load in HIV-1 infection. Nature. 1997;387(6629):183-188.

51. Zhang L, et al. Quantifying residual HIV-1 replication in patients receiving combination antiretroviral therapy. N Engl J Med. 1999;340(21):1605-1613.

52. Ramratnam B, et al. The decay of the latent reservoir of replication-competent HIV-1 is inversely correlated with the extent of residual viral replication during prolonged anti-retroviral therapy. Nat Med. 2000;6(1):82-85.
53. Finzi $\mathrm{D}$, et al. Latent infection of $\mathrm{CD} 4^{+} \mathrm{T}$ cells provides a mechanism of lifelong persistence of HIV-1, even in patients on effective combination therapy. Nat Med.1999;5(5):512-517.

54. Crooks AM, et al. Precise quantitation of the latent HIV-1 reservoir: implications for eradication strategies. JInfect Dis. 2015;212(9):1361-1365.

55. Dinoso JB, et al. Treatment intensification does not reduce residual HIV-1 viremia in patients on highly active antiretroviral therapy. Proc Natl Acad Sci U S A. 2009;106(23):9403-9408.

56. Gandhi RT, et al. The effect of raltegravir intensification on low-level residual viremia in HIV-infected patients on antiretroviral therapy: a randomized controlled trial. PLoS Med. 2010;7(8):e1000321.

57. Deeks SG. HIV: Shock and kill. Nature. 2012;487(7408):439-440.

58. Chun TW, et al. Effect of interleukin-2 on the pool of latently infected, resting CD4+ $T$ cells in HIV-1-infected patients receiving highly active anti-retroviral therapy. Nat Med. 1999;5(6):651-655.

59. Lafeuillade A, et al. Pilot study of a combination of highly active antiretroviral therapy and cytokines to induce HIV-1 remission. J Acquir Immune Defic Syndr. 2001;26(1):44-55.

60. Stellbrink HJ, et al. Effects of interleukin-2 plus highly active antiretroviral therapy on HIV-1 replication and proviral DNA (COSMIC trial). AIDS . 2002;16(11):1479-1487.

61. Archin NM, et al. Expression of latent human immunodeficiency type 1 is induced by novel and selective histone deacetylase inhibitors. AIDS. 2009;23(14):1799-1806.

62. Contreras X, et al. Suberoylanilide hydroxamic acid reactivates HIV from latently infected cells. J Biol Chem. 2009;284(11):6782-6789.

63. Archin NM, et al. Administration of vorinostat disrupts HIV-1 latency in patients on antiretroviral therapy. Nature. 2012;487(7408):482-485.

64. Elliott JH, et al. Activation of HIV transcription with short-course vorinostat in HIV-infected patients on suppressive antiretroviral therapy. PLoS Pathog. 2014;10(11):e1004473.

65. Rasmussen TA, et al. Panobinostat, a histone deacetylase inhibitor, for latent-virus reactivation in HIV-infected patients on suppressive antiretroviral therapy: a phase $1 / 2$, single group, clinical trial. Lancet HIV. 2014;1(1):e13-e21.

66. Archin NM, et al. HIV-1 expression within resting $\mathrm{CD}^{+}{ }^{+} \mathrm{T}$ cells after multiple doses of vorinostat. J Infect Dis. 2014;210(5):728-735.

67. Shan L, et al. Stimulation of HIV-1-specific cytolytic $\mathrm{T}$ lymphocytes facilitates elimination of latent viral reservoir after virus reactivation. Immunity. 2012;36(3):491-501.

68. Cillo AR, et al. Quantification of HIV-1 latency reversal in resting $\mathrm{CD} 4^{+} \mathrm{T}$ cells from patients on suppressive antiretroviral therapy. Proc Natl Acad Sci U S A. 2014;111(19):7078-7083.

69. Blazkova J, et al. Effect of histone deacetylase inhibitors on HIV production in latently infected, resting $\mathrm{CD} 4(+) \mathrm{T}$ cells from infected individuals receiving effective antiretroviral therapy. J Infect Dis. 2012;206(5):765-769.

70. Deng K, et al. Broad CTL response is required to clear latent HIV-1 due to dominance of escape mutations. Nature. 2015;517(7534):381-385.

71. Jones RB, et al. Histone deacetylase inhibitors impair the elimination of HIV-infected cells by cytotoxic T-lymphocytes. PLoS Pathog. 2014;10(8):e1004287.

72. Euler Z, Ly SH, Hurton M, Tsibris AMN, Alter G. Eradication of HIV-1 Reservoirs With Antibody Mediated Killing. Presented at: Conference on Retroviruses and Opportunistic Infections (CROI 2015); February 23-26, 2015; Seattle, Washington, USA. Abstract 426 .

73. Wei DG, et al. Histone deacetylase inhibitor romidepsin induces HIV expression in CD4 T cells from patients on suppressive antiretroviral therapy at concentrations achieved by clinical dosing. PLoS Pathog. 2014;10(4):e1004071.

74. Laird GM, et al. Ex vivo analysis identifies effective HIV-1 latency-reversing drug combinations. J Clin Invest. 2015;125(5):1901-1912.

75. Whitney JB, et al. Treatment with a TLR7 agonist induces transient viremia in SIV-infected ARTsuppressed monkeys. Presented at: Conference on Retroviruses and Opportunistic Infections (CROI 2015); February 23-26, 2015; Seattle, Washington, USA. Abstract 108.

76. Søgaard, OS, et al. The HDAC inhibitor romidep$\sin$ is safe and effectively reverses HIV-1 latency in vivo as measured by standard clinical assays. Presented at: 20th International AIDS Conference; July 20-25, 2014; Melbourne, Australia. Abstract OA2-4 LB.

77. Marsden MD, Zack JA. Neutralizing the HIV reservoir. Cell. 2014;158(5):971-972.

78. Dar RD, Hosmane NN, Arkin MR, Siliciano RF, Weinberger LS. Screening for noise in gene expression identifies drug synergies. Science. 2014;344(6190):1392-1396.

79. Kovochich M, Marsden MD, Zack JA. Activation of latent HIV using drug-loaded nanoparticles. PLoS One. 2011;6(4):e18270.

80. Veazey RS, et al. Gastrointestinal tract as a major site of $\mathrm{CD}^{+} \mathrm{T}$ cell depletion and viral replication in SIV infection. Science. 1998;280(5362):427-431.

81. Li Q, et al. Peak SIV replication in resting memory $\mathrm{CD} 4^{+} \mathrm{T}$ cells depletes gut lamina propria $\mathrm{CD} 4^{+} \mathrm{T}$ cells. Nature. 2005;434(7037):1148-1152.

82. Sigal A, et al. Cell-to-cell spread of HIV permits ongoing replication despite antiretroviral therapy. Nature. 2011;477(7362):95-98.

83. Abela IA, et al. Cell-cell transmission enables HIV-1 to evade inhibition by potent CD4bs directed antibodies. PLoS Pathog. 2012;8(4):e1002634.

84. Malbec M, et al. Broadly neutralizing antibodies that inhibit HIV-1 cell to cell transmission. J Exp Med. 2013;210(13):2813-2821.

85. Fukazawa Y, et al. B cell follicle sanctuary permits persistent productive simian immunodeficiency virus infection in elite controllers. Nat Med. 2015;21(2):132-139.

86. Kaschka WP, Theilkaes L, Eickhoff K, Skvaril F. Disproportionate elevation of the immunoglobulin G1 concentration in cerebrospinal fluids of patients with multiple sclerosis. Infect Immun. 1979;26(3):933-941.

87. Rubenstein JL. Rituximab therapy for CNS lymphomas: targeting the leptomeningeal compart- 
ment. Blood. 2002;101(2):466-468.

88. Balazs AB, et al. Vectored immunoprophylaxis protects humanized mice from mucosal HIV transmission. Nat Med. 2014;20(3):296-300.

89. Johnson PR, et al. Vector-mediated gene transfer engenders long-lived neutralizing activity and protection against SIV infection in monkeys. Nat Med. 2009;15(8):901-906.

90. Balazs AB, Chen J, Hong CM, Rao DS, Yang L, Baltimore D. Antibody-based protection against HIV infection by vectored immunoprophylaxis.
Nature. 2011;481(7379):81-84.

91. Ko SY, et al. Enhanced neonatal Fc receptor function improves protection against primate SHIV infection. Nature. 2014;514(7524):642-645.

92. Diskin R, et al. Increasing the potency and breadth of an HIV antibody by using structure-based rational design. Science. 2011;334(6060):1289-1293.

93. Diskin R, et al. Restricting HIV-1 pathways for escape using rationally designed anti-HIV-1 antibodies. J Exp Med. 2013;210 (6):1235-1249.
94. Galimidi RP, et al. Intra-spike crosslinking overcomes antibody evasion by HIV-1. Cell. 2015;160(3):433-446.

95. Pace CS, et al. Bispecific antibodies directed to $\mathrm{CD} 4$ domain 2 and HIV envelope exhibit exceptional breadth and picomolar potency against HIV-1. Proc Natl Acad Sci US A. 2013;110(33):13540-13545.

96. Denton PW, et al. Targeted cytotoxic therapy kills persisting HIV infected cells during ART. PLoS Pathog. 2014;10(1):e1003872. 